\title{
Optogenetic Analysis of Neuronal Excitability during Global Ischemia Reveals Selective Deficits in Sensory Processing following Reperfusion in Mouse Cortex
}

\author{
Shangbin Chen, Majid H. Mohajerani, Yicheng Xie, and Timothy H. Murphy \\ Department of Psychiatry, Brain Research Centre, University of British Columbia, Vancouver, British Columbia V6T 1Z3, Canada
}

We have developed an approach to directly probe neuronal excitability during the period beginning with induction of global ischemia and extending after reperfusion using transgenic mice expressing channelrhodopsin-2 (ChR2) to activate deep layer cortical neurons independent of synaptic or sensory stimulation. Spontaneous, ChR2, or forepaw stimulation-evoked electroencephalogram (EEG) or local field potential (LFP) records were collected from the somatosensory cortex. Within $20 \mathrm{~s}$ of ischemia, a $>90 \%$ depression of spontaneous $0.3-3 \mathrm{~Hz}$ EEG and LFP power was detected. Ischemic depolarization followed EEG depression with a $\sim 2$ min delay. Surprisingly, neuronal excitability, as assessed by the ChR2-mediated EEG response, was intact during the period of strong spontaneous EEG suppression and actually increased before ischemic depolarization. In contrast, a decrease in the somatosensory-evoked potential (forepaw-evoked potential, reflecting cortical synaptic transmission) was coincident with the EEG suppression. After $5 \mathrm{~min}$ of ischemia, the animal was reperfused, and the ChR2-mediated response mostly recovered within $30 \mathrm{~min}$ ( $>80 \%$ of preischemia value). However, the recovery of the somatosensory-evoked potential was significantly delayed compared with the ChR2-mediated response $(<40 \%$ of preischemia value at $60 \mathrm{~min}$ ). By assessing intrinsic optical signals in combination with EEG, we found that neuronal excitability approached minimal values when the spreading ischemic depolarization wave propagated to the ChR2-stimulated cortex. Our results indicate that the ChR2mediated EEG/LFP response recovers much faster than sensory-evoked EEG/LFP activity in vivo following ischemia and reperfusion, defining a period where excitable but synaptically silent neurons are present.

\section{Introduction}

Stroke is a major cause of death and disability that results from a transient or permanent reduction in cerebral blood flow (Dirnagl et al., 1999; Hossmann, 2006; Murphy and Corbett, 2009). During stroke, neuronal structure and function are rapidly damaged (Risher et al., 2010; Mostany and PorteraCailliau, 2011), but can in part recover during reperfusion (Zhang et al., 2005; Li and Murphy, 2008; Murphy et al., 2008). Although structure can recover after reperfusion, brain activity is depressed as measured by spontaneous cortical electroencephalogram (EEG) activity (Murphy et al., 2008) or somatosensory-evoked potential (Astrup et al., 1981; Lesnick et al., 1986). It is unclear whether this prolonged depression is due to a lack of a hyperpolarized membrane potential ( $\mathrm{Xu}$ and Pulsinelli, 1994) or a defect that selectively impairs excitability

Received March 23, 2012; revised June 26, 2012; accepted July 21, 2012.

Author contributions: S.C. and T.H.M. designed research; S.C. and Y.X. performed research;S.C., M.H.M., and Y.X. analyzed data; S.C. and T.H.M. wrote the paper.

This work was supported by a grant-in-aid from the Heart and Stroke Foundation of British Columbia and the Yukon, a Canadian Institutes of Health Research Grant MOP 111009, and a Human Frontier Science Program grant to T.H.M. We thank Cindy Jiang and Pumin Wang for surgical assistance; Jeff LeDue and Jamie Boyd for assistance with the experimental system; and Oliver G. S. Ayling, Sherri Tran, and Thomas C. Harrison for constructive suggestions and sharing preliminary data.

The authors declare no competing financial interests.

Correspondence should be addressed to Dr. Timothy H. Murphy, 4N1-2255 Wesbrook Mall, University of British Columbia, Vancouver, BC V6T 123, Canada. E-mail: thmurphy@mail.ubc.ca.

DOI:10.1523/JNEUROSCI.1439-12.2012

Copyright $\odot 2012$ the authors $\quad 0270-6474 / 12 / 3213510-10 \$ 15.00 / 0$ or synaptic transmission (Gao et al., 1998). Since ischemia can affect upstream sensory or presynaptic pathways (Pang et al., 2002; Li et al., 2009), or enhance inhibition (Clarkson et al., 2010), sensation is not always a reliable means for assessing excitability. Electrophysiological measurements involving direct antidromic neuronal stimulation may be difficult to employ and interpret in vivo. Here, we selectively examine neuronal excitability during the period beginning with the induction of ischemia and extending after reperfusion using transgenic mice [B6.Cg- $\mathrm{Tg}$ (Thy1COP4/EYFP)18Gfng/J] expressing channelrhodopsin-2 (ChR2) in predominantly layer 5 cortical pyramidal neurons (Arenkiel et al., 2007; Wang et al., 2007; Ayling et al., 2009). ChR2 is a directly light-gated nonselective cation channel, which when expressed in neurons can transduce light energy into neural activity (Nagel et al., 2003; Boyden et al., 2005; Miesenböck, 2009). With conventional EEG and multichannel local field potential (LFP) as readouts, the ChR2-based technique can optically interrogate defined circuit elements with millisecond-scale, and cell type-specific precision in vivo (Zhang et al., 2007). This optogenetic approach has the advantage of being able to directly assess neuronal excitability independently of changes to synaptic activity. In addition, we use a mouse in vivo global ischemia model that allows us to titrate the duration of ischemia to within seconds and can rapidly alternate between the initiation of ischemia or reperfusion (Murphy et al., 2008; Liu and Murphy, 2009). Using this approach within the sensorimotor cortex, we show that prolonged postischemic depression of sensory-evoked activity is not attributed to a loss of 
A

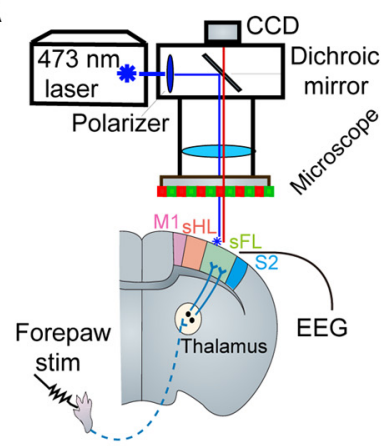

ChR2-YFP expression

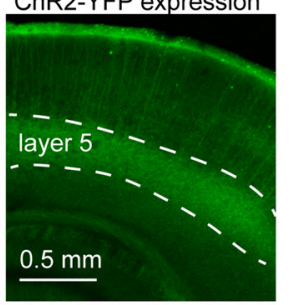

B

ChR2 or sensory stimulation, EEG recording

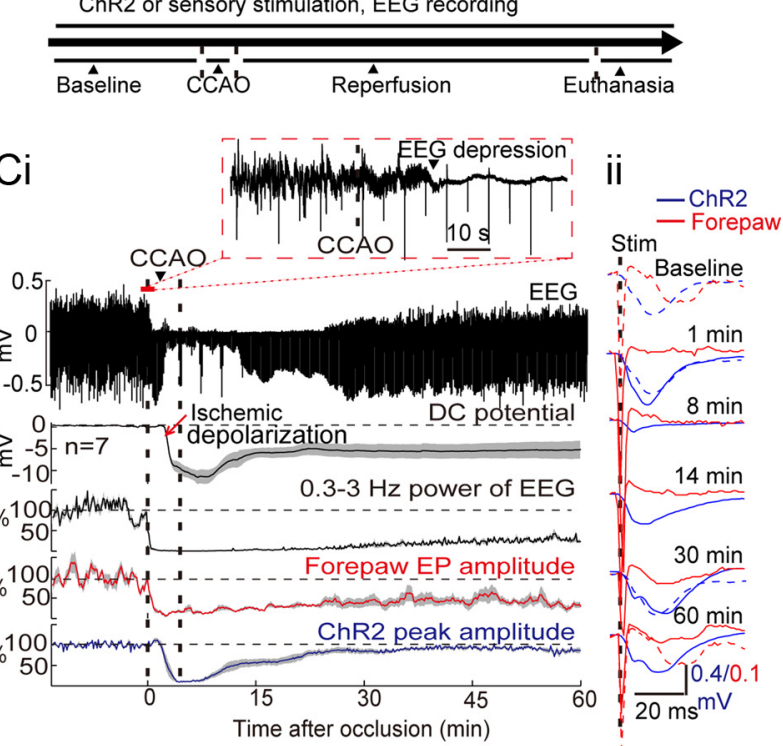

E - Forepaw

- ChR2

Phase 1

Phase 2

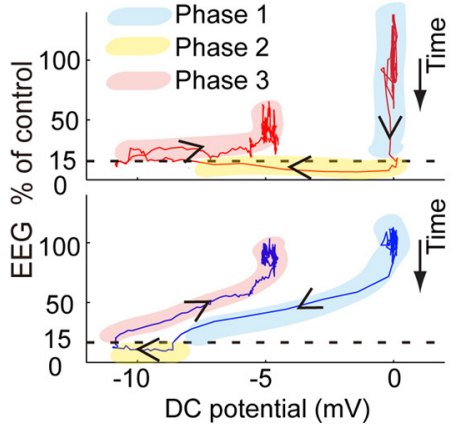

Figure 1. EEG recording reveals selective deficits in sensory processing and rapid recovery of ChR2 response following reperfusion. $A$, Schematic drawing of experimental system. EEG recording, IOS imaging, photostimulation, and electrical forepaw stimulation were coordinated with synchronized trigger signals. ChR2 and sensory stimulation were interleaved and repeated every $10 \mathrm{~s}$. Coronal brain slice indicates that ChR2 is predominantly expressed in layer 5 of cortex. $B$, Experimental timeline: ChR2 and sensory stimulation were performed during baseline, CCA0, reperfusion, and after the animal was killed ( $\sim 3 \mathrm{~h}$ for the timeline). Ci, Changes of spontaneous EEG (example mouse), DC potential ( $n=7$ mice), $0.3-3 \mathrm{~Hz}$ EEG power ( $n=7$ mice), forepaw-evoked potential (EP) ( $n=7$ mice), and ChR2 peak amplitude ( $n=7$ mice) are shown from top to bottom. In the EEG record, an enlarged view shows EEG depression within $20 \mathrm{~s}$ after CCA0 (inset). Ischemic depolarization is indicated with an arrow over the DC potential record. Except for EEG and DC potential, the other statistical data were based on relative values, which were normalized with baseline level as $100 \%$. The shaded band indicates SEM. Spontaneous EEG power and forepaw-evoked EEG response were depressed immediately after CCA0 and recovered relatively slowly. However, ChR2 peak amplitude is transiently increased 1-2 min after occlusion, followed by a decrease in response and relatively rapid recovery with reperfusion. Cii, Six time segments of ChR2-/forepaw-evoked EEG data. The vertical dashed line indicates the onset of ChR2 or forepaw stimulation (a stimulus artifact is present during forepaw stimulation). The baseline responses of ChR2/forepaw stimulation are shown with dashed lines and superimposed over selected records. ChR2-mediated response shows a larger amplitude than the baseline (blue dash line) at 1 min after CCAO (trace 2), and relatively faster recovery at $30 \mathrm{~min}$ after CCA0. The forepaw-evoked potential shows prolonged suppression at $60 \mathrm{~min}$ after CCAO compared with the baseline response (red dash line). $\boldsymbol{D}$, Statistical analysis of $0.3-3 \mathrm{~Hz}$ EEG power, forepaw-, or ChR2-mediated EEG responses during global ischemia at select time points. ${ }^{*} p<0.05$; ${ }^{* *} p<0.01$. E, Forepaw/ChR2-mediated EEG responses are shown in phase plots for comparison to DC potential ( $n=7$ mice). By setting a 15\% level of excitable response as a threshold, a three-phase relationship was observed: phase 1 (shadowed with cyan), initiation of ischemia and suppression of response to $15 \%$ of baseline; phase 2 (shadowed with yellow), maximum inhibition; phase 3 (shadowed with pink), partial recovery of DC potential and excitable response. The primary motor cortex (M1) and hindlimb (SHL), forelimb somatosensory cortex (SFL), and secondary somatosensory cortex (S2) are indicated. Stim, Stimulation.

neuronal excitability since ChR2-mediated EEG responses are recovered following reperfusion.

\section{Materials and Methods}

Transgenic mice. We have studied a total of 24 adult male Thy1-ChR2yellow fluorescent protein (YFP) transgenic mice [B6.Cg- $\mathrm{Tg}(T h y 1-$ COP4/EYFP)18Gfng/J; Fig. 1 A, ChR2-YFP expression) (Arenkiel et al., 2007), which were obtained from Jackson Laboratories and bred at the University of British Columbia animal facilities. The mice were 2-4 months of age and ranged in weight between 26 and $32 \mathrm{~g}$. All experiments used urethane anesthesia, as in Murphy et al., (2008). Body temperature was maintained at $37 \pm 0.5^{\circ} \mathrm{C}$ using a heating pad and feedback regulation from a rectal temperature probe (FHC). Hydration was maintained by intraperitoneal injection of saline (200-300 $\mu \mathrm{l})$ with $20 \mathrm{~mm}$ glucose at $1-2 \mathrm{~h}$ intervals. The experimental protocols were approved by the University of British Columbia Animal Care Committee and were consistent with the Canadian Council on Animal Care and Use guidelines.

Surgical procedures. The surgical procedures for preparing a cranial window and the in vivo intrinsic optical signals (IOSs) imaging methods have been described previously (Zhang et al., 2005). Briefly, animals were fitted into a custom-made head holder. A stainless steel chamber that covered mainly the right parietal cortex was adhered to the skull with ethyl cyanoacrylate (Krazy Glue, Elmer's Products). The animal was then prepared for a global ischemia model (see details in Murphy et al., 2008). A $4 \times 4 \mathrm{~mm}$ cranial window was made over the somatosensory cortex, leaving the dura intact. The cortex was covered with $1.3 \%$ low-melt 
agarose (at $37-38^{\circ} \mathrm{C}$; Type 3-A; A9793; Sigma) dissolved in HEPESbuffered artificial CSF (ACSF) and sealed with a glass coverslip (\#1). For optrode recording (described below), a smaller cranial window $(2 \times 2$ $\mathrm{mm}$ ) was made to cover the forelimb cortex with ACSF filling a well made of dental cement without a coverslip. A tracheotomy was performed on all animals to alleviate breathing problems, which may have been confounded by surgical manipulation of the tissue within the neck region or prolonged urethane anesthesia (Li and Murphy, 2008). After surgery, the animal was allowed to recover (under anesthesia) for at least $30 \mathrm{~min}$ before further experimental recording.

Global ischemia model. The common carotid arteries (CCAs) were exposed bilaterally and sutures (5-0, silk; Ethicon) were looped around each CCA. The animal was turned upright, placed in a head holder, and the craniotomy procedure performed. To induce global ischemia, we carefully pulled on both sutures and secured them with tape to induce bilateral CCA occlusion (CCAO). Ischemia was induced 15-40 min after EEG recording, and IOS imaging (or optrode recording) was begun. For experiments using EEG recordings with forepaw or ChR2 stimulation, only animals exhibiting $>8 \mathrm{mV}$ of DC shift were considered as successful global ischemia models. To avoid compromising the health of the animals, we limited the ischemic period to $\sim 5 \mathrm{~min}$, and animals were reperfused by cutting the sutures. After $1-1.5 \mathrm{~h}$ of reperfusion, the animal was killed with an intraperitoneal injection of Euthanyl $(0.10 \mathrm{ml})$ (Figure $1 B$, timeline).

ChR2 stimulation. $\mathrm{f}$ (CNI Optoelectronics) was used to stimulate ChR2expressing neurons. The relatively collimated laser beam was delivered to the brain surface via an Olympus BX51 upright microscope, and the diameter measured through the objective $(4 \times)$ was $43 \mu \mathrm{m}$ at the brain surface (Fig. $1 A, n=7$ mice). The beam was positioned over the somatosensory cortex as determined by stereotaxic coordinates. Laser output $\sim 1 \mathrm{~mW}$ premeasured with a power meter (Thorlabs) was triggered by a transistor-transistor logic pulse of 5 ms duration for each photostimulation (Arenkiel et al., 2007; Ayling et al., 2009). The power was $\sim 700 \mathrm{~mW} / \mathrm{mm}^{2}$ at brain surface. Based on previous work addressing light spreading and scattering in brain tissue (Aravanis et al., 2007; Kahn et al., 2011), the laser power at the depth of layer 5 (800 $\mu \mathrm{m}$, where most ChR2-YFP-labeled neuronal cell bodies were found) was expected to be $\sim 6 \mathrm{~mW} / \mathrm{mm}^{2}$; however, light levels at superficial dendrites were likely much higher. Laser power for ChR2 stimulation was adjusted (1-5 mW) following one to two pilot trials to obtain a $\geq 0.5 \mathrm{mV}$ peak EEG response, which was measured with a surface electrode.

Forepaw stimulation. Two metal pins were inserted into the left forepaw, and a $1-5 \mathrm{~mA} 1$ or $2 \mathrm{~ms}$ electrical pulse was delivered. The electrical pulse was generated by a constant current stimulus isolator (A385, World Precision Instruments) triggered $5 \mathrm{~s}$ after ChR2 laser stimulation by a 1322A Digidata system (Molecular Devices).

EEG recording. For EEG recording, a Teflon-coated chlorided silver wire (125 $\mu \mathrm{m}$; World Precision Instruments) with an exposed tip $(\sim 1$ $\mathrm{mm}$ ) was placed on the cortical surface within the agarose. For EEG recording combined with optrode recording, the EEG electrode was placed in the ACSF and the reference electrode was placed on the nasal bone under the skin (Murphy et al., 2008; Mohajerani et al., 2010). The cortical signal was amplified and filtered (gain $100 \times, 0-1000 \mathrm{~Hz}$ ) using a differential amplifier (DAM50, World Precision Instruments) and digitized $(1000 \mathrm{~Hz})$ using the 1322A Digidata. The output from the DAM50 amplifier was also sent to a USB-based device (MiniDigi 1A, Molecular Devices) then acquired by an Axoscope 9.2 (Molecular Devices) for continuous recording. In our experiment, Clampex software was used as the central controller for timing the experimental system.

Optrode recording. To obtain information from different cortical depths, a silicon optrode (A1x16-3 mm-50-413-Op16, NeuroNexus Technologies) was used for recording LFP and multiunit activity (MUA) and ChR2 stimulation in eight mice (Kravitz et al., 2010; Kahn et al., 2011). The optrode contained $16413 \mu \mathrm{m}^{2}$ recording sites (0.7-1.2 $\mathrm{M} \Omega$ impedance at $1 \mathrm{kHz}$ ), arranged linearly with $50 \mu \mathrm{m}$ spacing between each site. A $473 \mathrm{~nm}$ laser (IKE-473-100-OP, IkeCool) was coupled to the optrode using a fiberoptic patch cord (FC-x.x-NNC, NeuroNexus Technologies). Based on the atlas of mouse cortex from the Allen Institute for Brain Science, the optrode probe was inserted into the forelimb somatosensory cortex to a depth of $800 \mu \mathrm{m}$ (bregma $0.3 \mathrm{~mm}$, lateral $2.0 \mathrm{~mm}$ ) to target layers 1-5. Here, ChR2 stimulation was performed with an optrode-integrated laser fiber (105 $\mu \mathrm{m}$ diameter). The fiber tip was positioned $\sim 50 \mu \mathrm{m}$ above the uppermost recording site near the cortical surface. Based on previous work with a similar probe (Kravitz et al., 2010), laser power was adjusted to yield $0.5-2 \mathrm{~mW}$ at the fiber tip to obtain clear LFP deflections from the deepest recording site. In one preliminary experiment, laser power of $0.5-5 \mathrm{~mW}$ was systematically varied. The quantified LFP deflection showed a hyperbolic tangential increase with laser power (data not shown). By fitting to the tangential function, $0.5-2 \mathrm{~mW}$ laser power was found to evoke $72-99 \%$ of the maximum response. LFP recordings were performed from the optrode using a portable 16-channel data acquisition system (USB-ME16-FAI-System, Multi Channel Systems). After 1000× amplification, all 16-channel LFP signals were sampled at $25 \mathrm{kHz}$ with a bandwidth of 1-5000 Hz.

Pharmacology. Seven ChR2 mice were used for pharmacological experiments. For experiments $(n=4)$ involving glutamate receptor antagonists, DNQX $(200 \mu \mathrm{M})$ and APV $(500 \mu \mathrm{M})$ (Sigma) were dissolved in ACSF and applied to the craniectomy. Experiments $(n=3)$ using tetrodotoxin (TTX; $30 \mu \mathrm{M}$, Sigma) used local microinjection within the craniotomy, and were performed under ketamine-xylazine anesthesia using a similar collimated laser delivery system (Ayling et al., 2009) with $10 \mathrm{~ms}$ laser pulses.

Electrophysiology analysis. Since CCAO caused a DC shift during the recording (Murphy et al., 2008; Tran et al., 2012), linear fitting was used to remove the shifting baseline of EEG signal. The continuous EEG recording from the Axoscope was performed routinely with off-line highpass filtering $(0.1 \mathrm{~Hz}$, Bessel filter, 8 pole $)$ to exclude the slow DC shift. EEG power of $0.3-3 \mathrm{~Hz}$ was calculated from the filtered EEG signal (only $4.5 \mathrm{~s}$ of data between ChR2 and forepaw stimulation in each period were used to avoid stimulation interference) with the frequency domain filtered to $0.3-3 \mathrm{~Hz}$ (Murphy et al., 2008). The amplitude from ChR2 stimulation was defined as the minimum EEG signal within $40 \mathrm{~ms}$ after onset of ChR2 stimulation. To reduce the photoelectric artifact (Ayling et al., 2009), the ChR2 responses were corrected by subtracting ChR2 responses obtained $10 \mathrm{~min}$ after the death of the animal. For forepaw electrical stimulation, the evoked potential was extracted from linear corrected EEG data by determining the minimum value within $20 \mathrm{~ms}$ after stimulation. Forepaw-evoked potentials were averaged over $1 \mathrm{~min}$. Only the amplitude of the large primary cortical wave was determined (Lesnick et al., 1986), which corresponded to the N1 wave (Fujioka et al., 2004).

For 16-channel optrode recording, $50 \mathrm{~ms}$ columnar ChR2 and sensory-evoked LFP responses were segmented to display data from baseline, during occlusion, and after reperfusion. To quantify the ChR2mediated LFP responses, the minimum voltage value of $8-12 \mathrm{~ms}$ after the ChR2 stimulation onset was extracted. For sensory-evoked LFP responses, the minimum of $10-20 \mathrm{~ms}$ after the forepaw stimulation onset was calculated. Since the bandwidth of LFP recording was $1-5000 \mathrm{~Hz}$, the mean power of $1-3 \mathrm{~Hz}$ was calculated as previously (Mohajerani et al., 2010). Only LFP data from $500 \mu \mathrm{m}$ depth (beginning in layer 5) were quantified for group data. To resolve spikes from LFP recording, off-line bandpass filtering (600-6000 Hz, elliptic filter) was performed to acquire MUA (Kahn et al., 2011). Automated amplitude thresholding (four times the SD of the background noise) was used to detect spikes from the filtered data (Quiroga et al., 2004). Relatively large laser stimulation artifacts were removed by setting another upper-bound threshold (40 times SD). To reduce possible false-positive spikes, a principal component analysis was used to extract features of spike shapes that were used for excluding outliers (Bokil et al., 2010). The selected spikes were presented in a raster plot. The open source program Wave Clus (Quiroga et al., 2004) was used for this analysis.

IOS imaging. For IOS imaging, the cortical surface was illuminated by red light-emitting diodes (center at $635 \mathrm{~nm}$ ) mounted around a microscope objective (Harrison et al., 2009). Imaging was performed with a charge-coupled device (CCD) camera (1M60 Pantera, Teledyne DALSA), and EPIX E4DB frame grabber. The 12-bit CCD camera used for IOS was mounted on an Olympus BX51 upright scope with a $4 \times, 0.13$ numerical aperture UPlan Fl Olympus objective. We typically imaged an area of $3.2 \times 3.2 \mathrm{~mm}$ using a $4 \times 4$ pixel binning mode (i.e., 
$256 \times 256$ pixels for one single image, with a pixel size of $12.5 \times 12.5$ $\mu \mathrm{m})$. IOS imaging was not performed during optrode recording experiments.

Image analysis. To visualize the ischemic depolarization waves, a differential image method for processing the image sequence was performed as in previous work (Chen et al., 2006a,b). Within an image sequence, we selected two small regions of interest (ROIs) $(5 \times 5$ pixel size) located in the cortical parenchyma. The average reflectance intensity in the ROI of each frame was calculated. Choosing the reflectance before ischemic depolarization onset as the baseline value, we calculated the relative changes of reflectance during an ischemic depolarization event.

Statistics. In the 17 animal experiments for global ischemia, 15 of the animals permitted successful recording of both consistent ChR2 and forepaw stimulation responses ( $\mathrm{DC}$ shift $>8 \mathrm{mV}$ ). Two of the 17 animals did not show a typical DC shift after CCAO. Only data from 15 animals that produced clear DC shifts were used for statistical analysis. Except for DC potential, the other statistical variables (e.g., EEG power and ChR2 peak amplitude) were based on normalized relative values. The mean baseline level values before CCAO $15 \mathrm{~min}$ from each animal were used to normalize the absolute measurements. All group data were expressed as the mean \pm SEM. Phase plots were created by plotting the mean values for forepaw and ChR2-mediated EEG amplitude on the $y$-axis and DC potential on the $x$-axis for each point in time (Ba et al., 2002). A Student's $t$ test was used for testing whether the group data showed significant changes at different time points relative to the baseline level 100\%. Values were considered statistically significant at $p<0.05$.

\section{Results \\ EEG analysis of cortical excitability during global ischemia using ChR2}

Here we adapt a mouse model of global ischemia (Murphy et al., 2008; Liu and Murphy, 2009; Tran et al., 2012) for direct optogenetic activation of subpopulations of cortical neurons from B6.Cg-Tg(Thy1-COP4/EYFP)18Gfng/J ChR2 transgenic mice before, during, and after global ischemia. These mice express ChR2 in predominantly cortical layer 5 (Arenkiel et al., 2007; Wang et al., 2007; Ayling et al., 2009; Kahn et al., 2011) (Fig. 1 A). Given the diffuse labeling that is also present, we cannot rule out that a minority of expression occurs in other layers. The global ischemia model permits the induction of ischemia and reperfusion while retaining access to the cortical surface for light stimulation of ChR2 or imaging (Murphy, 2011) (Fig. 1A). ChR2 was used to assess neuronal excitability independent of sensory stimulation every $10 \mathrm{~s}$ during baseline, ischemia, and reperfusion in combination with electrophysiological methods. During periods of baseline activity in preischemic animals, we observed a regular EEG signal consisting of $\sim 0.3-3 \mathrm{~Hz}$ activity, which is expected with urethane anesthesia (Crunelli and Hughes, 2010; Mohajerani et al., 2010; Barth and Mody, 2011). Bilateral CCAO ( 5 $\mathrm{min})$ resulted in a rapid suppression of EEG activity within $20 \mathrm{~s}$, consistent with previous reports (Murphy et al., 2008) ( $n=7$ mice; Fig. 1Ci). Following the EEG depression with a $\sim 2 \mathrm{~min}$ delay, an ischemic depolarization characterized by a shift in DC potential was observed (amplitude: $\sim 8-18 \mathrm{mV}$; duration: 7-10 min) (Fig. 1Ci). There was a small transient hyperpolarization (1-2 $\mathrm{mV}$ ) of DC potential before the negative DC potential shift (depolarization), consistent with previous work (Xu and Pulsinelli, 1994). Given these robust EEG changes, the loss of a normal cortical EEG was used as a marker of ischemic onset. Following reperfusion, the DC potential partially recovered within $10 \mathrm{~min}$ (to $-5.7 \pm 1.2 \mathrm{mV}, n=7$ ) (Fig. 1Ci). However, spontaneous EEG power quantified over $0.3-3 \mathrm{~Hz}$ showed relatively slower recovery. Even $1 \mathrm{~h}$ after CCAO, EEG power was $<40 \%$ of baseline levels.
While simultaneously monitoring the spontaneous EEG and forepaw sensory response, ChR2 stimulation was used to assess neuronal excitability by measuring the depolarizing (negative going) EEG response to light (Fig. 1Cii). Brief $5 \mathrm{~ms}$ pulses of $473 \mathrm{~nm}$ light produced a depolarizing EEG response that contained components expected from rapid (within 15-25 ms) direct ChR2 activation (Ayling et al., 2009), as well as delayed responses that may reflect secondary activation of neurons through synaptic transmission (Harrison et al., 2012; Scott and Murphy, 2012). During baseline EEG recordings (15-40 min before CCAO), the ChR2 response peak from each $5 \mathrm{~ms} 473 \mathrm{~nm}$ laser stimulation pulse was $0.5-2 \mathrm{mV}$ and occurred $\sim 15-25 \mathrm{~ms}$ after stimulation. Forepaw stimulation ( $1 \mathrm{~ms}$ electrical pulse)-evoked EEG responses had a peak amplitude of $\sim 0.05-0.10 \mathrm{mV}$ that occurred $\sim 15-20 \mathrm{~ms}$ after electrical stimulation in animals before ischemia. The peak ChR2-mediated EEG response was used as a measure of neuronal excitability. Within $1 \mathrm{~min}$ after occlusion, the forepaw-evoked response was blocked, while the ChR2-mediated response was preserved (Fig. 1Cii). Surprisingly, neuronal excitability as monitored by ChR2 and EEG was intact during the first 2 min of global ischemia despite strong suppression of spontaneous EEG activity. The ChR2-mediated EEG response actually increased $1 \mathrm{~min}$ following occlusion before ischemic depolarization (Fig. 1Cii). Both ChR2 and forepaw-evoked responses were markedly suppressed within $3 \mathrm{~min}$ of CCAO. During reperfusion after $5 \mathrm{~min}$ of ischemia, the ChR2mediated EEG response showed faster recovery than the forepaw-evoked response (Fig. 1D).

\section{Relationship between DC potential and excitability assessed using ChR2}

To examine the relationship between neuronal excitability (measured by forepaw- or ChR2-mediated responses) and ischemic depolarization, phase plots were constructed (Fig. 1E). Specifically, the phase plots were used to graphically display how changes in the ChR2-mediated or sensory-evoked EEG response were related to DC potential during ischemia and reperfusion in group data. By setting a $15 \%$ level of excitable response as a threshold, a three-phase relationship was observed: phase 1, initiation of ischemia and suppression of response to $15 \%$ of baseline; phase 2, maximum inhibition; and phase 3 , recovery during reperfusion. The forepaw-evoked EEG response showed a rapid decrease even before a change in DC potential was observed during phase 1, while the ChR2 measure of EEG excitability was relatively unaffected. Interestingly, during phase 1 the ChR2mediated EEG response was gradually attenuated in proportion to the loss of DC potential. The use of linear regression indicated that the loss of DC potential and the ChR2 response were strongly correlated $(r=0.93, p<0.01)$, while DC potential and forepaw response were not $(r=0.05, p>0.05)$. During phase $3, \mathrm{DC}$ potential gradually recovered and an approximately proportional return of the ChR2-mediated response was observed $(r=0.91$, $p<0.01)$. However, the recovery of the forepaw-evoked response during phase 3 was significantly delayed and incomplete despite the recovery of DC potential (Fig. $1 E$ ). The use of phase plots indicates that changes in excitability assessed by the ChR2mediated EEG response are strongly correlated with the DC potential, while the forepaw-evoked or spontaneous EEG response shows defects that precede the loss of DC potential and are still apparent even after the DC potential has appreciably recovered (Fig. 1E). 
$\mathrm{Ai}$

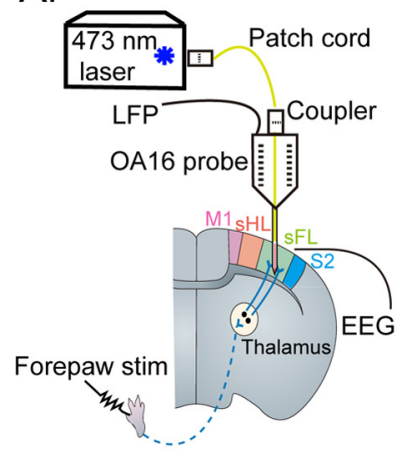

ii

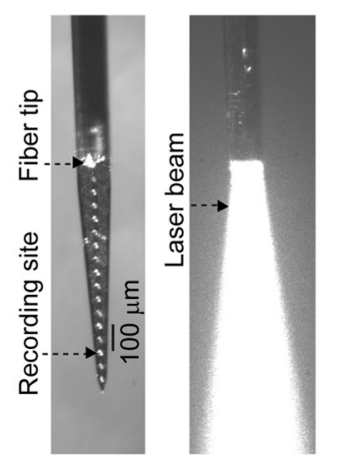

iii

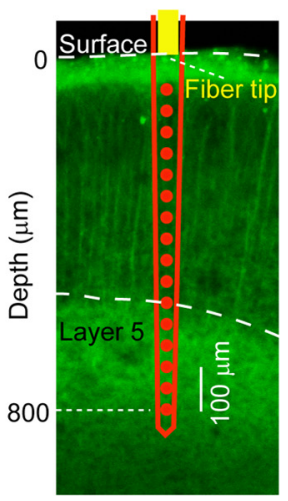

$\mathrm{Bi}$

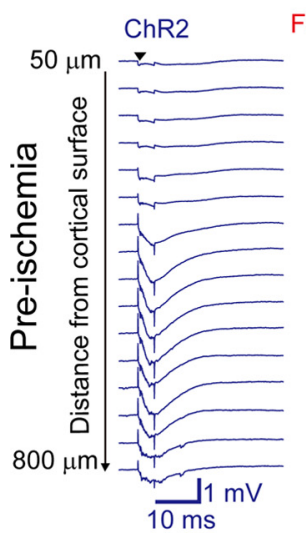

C

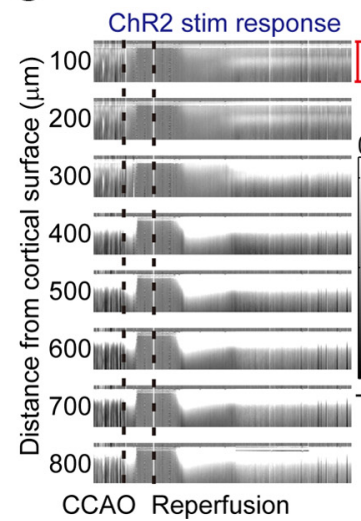

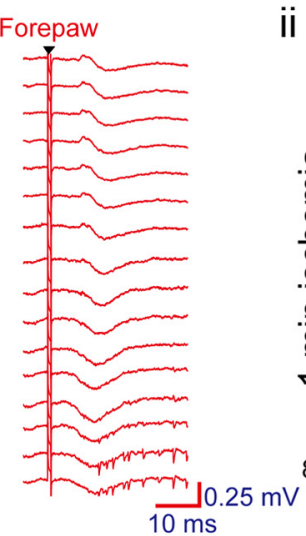

Forepaw stim response

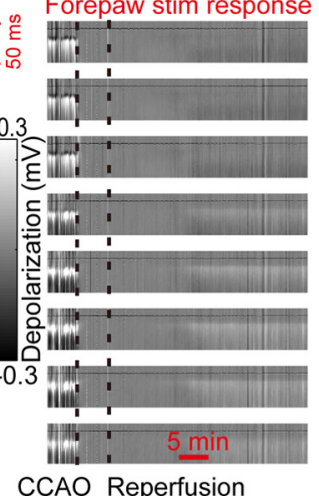

ii
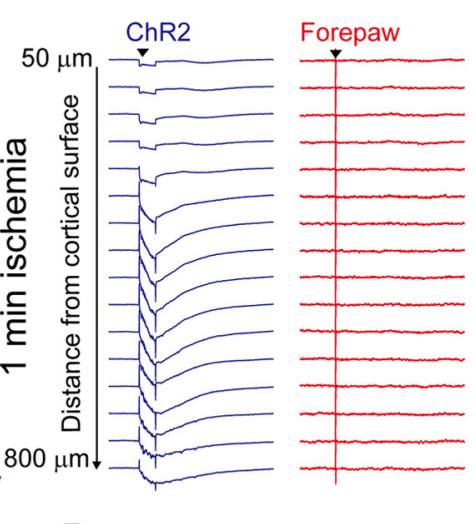

D

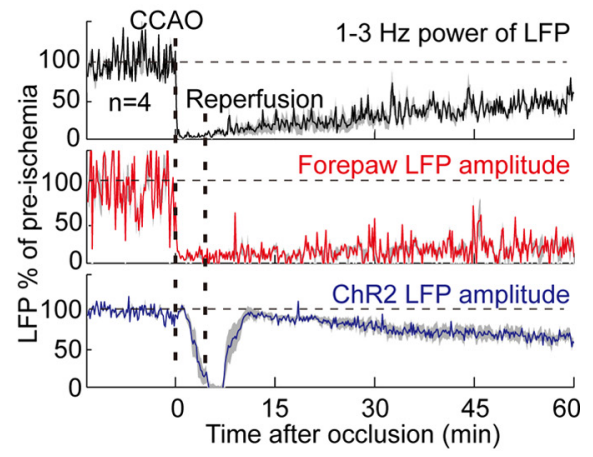

Figure 2. LFP-based electrophysiology confirms selectivedeficits in sensory processing after global ischemia.Ai,A 16-channel optrode (OA16,NeuroNexus Technologies) was usedfor LFP recording and ChR2 stimulation. Aii, Laser light exitsfrom the fibertip ( $\sim 50 \mu$ mabove the firstrecording site). Aiis shows thelaser distribution before implantation in cortical tissue. Aiii shows the approximate location of the optrode in cortical layers $1-5$. Bi, Bii, Representative LFP data from preischemia (Bi) and 1 min after ischemia (Bii) from cortical layers 1-5 (up to $800 \mu$ m depth from the brain surface). Compared with preischemia results, the data from 1 min after ischemia show preservation of ChR2-mediated response and a lack of forepaw-evoked response. C, ChR2-mediated LFP responses from 8 recording sites (half of all 16 sites) to a depth of $800 \mu \mathrm{m}$ below the cortical surface, which are visualized as $50 \mathrm{~ms}$ columnar time sweeps. Preserved ChR2 responses are seen in the period between CCAO and ischemic depolarization. ChR2 responses are suppressed at all measured sites following ischemic depolarization and recoverfaster indeeper layers compared with moresuperficial layers of cortex.Sensory responses aresuppressedfollowing CCAO and slowlyrecover during reperfusion. D, Group data of $1-3 \mathrm{HzL}$ LP power, forepaw-evoked LFP amplitude, and ChR2-mediated LFP amplitude. stim, Stimulation.

\section{LFP characterization of ChR2- and sensory-evoked responses during global ischemia}

Although surface EEG recording showed robust changes in both sensory and ChR2 responses following global ischemia, it was not clear whether these effects were present throughout the depth of cortex. Using a 16-channel optrode, we monitored spontaneous, sensory, and ChR2-mediated changes in LFP within cortical layers 1-5 (Fig. 2A). Before CCAO, we observed a robust spontaneous LFP signal, consisting primarily of relatively low-frequency $\sim 1-3 \mathrm{~Hz}$ activity. After $1 \mathrm{~min}$ of CCAO, the forepaw-evoked and spontaneous LFP signals were suppressed, but the ChR2mediated peak LFP response was preserved across cortical depth
(Fig. 2Bi,Bii). Analysis of ChR2-mediated response recovery (during reperfusion), using LFP sweeps that were stacked to form images, indicated that all depths exhibited relatively faster and more complete recovery than the forepaw-evoked response (Fig. $2 C)$. Since the $\mathrm{ChR} 2$ was expressed predominantly in layer 5 , we selected the LFP signal from the $500 \mu \mathrm{m}$ depth recording site to quantify the changes during global ischemia within the group data (Fig. 2D). Plotting the ChR2 response from layer 5 indicated nearly full recovery of the ChR2-mediated response after 7-10 min of reperfusion ( $>90 \%$ of baseline level), but only minimal recovery of the sensory-evoked LFP response (Fig. $2 D$ ). In additional longer term recording experiments $(n=4$ mice $)$ with 
A

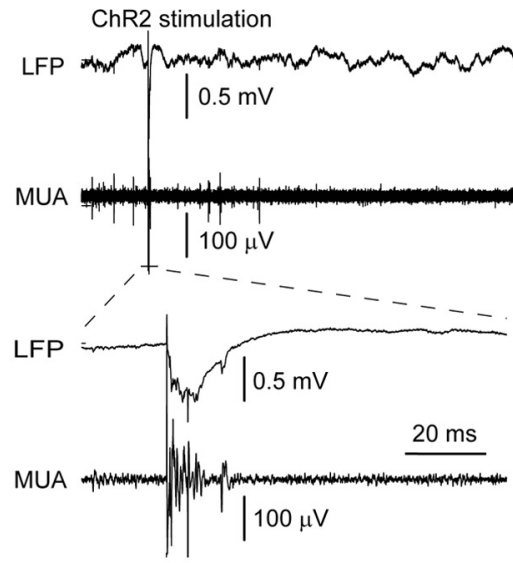

B
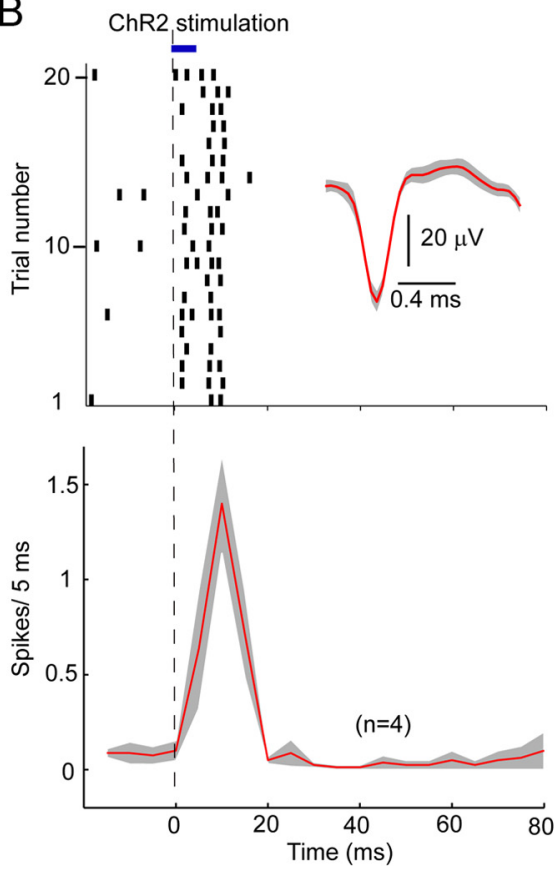

Forepaw stimulation

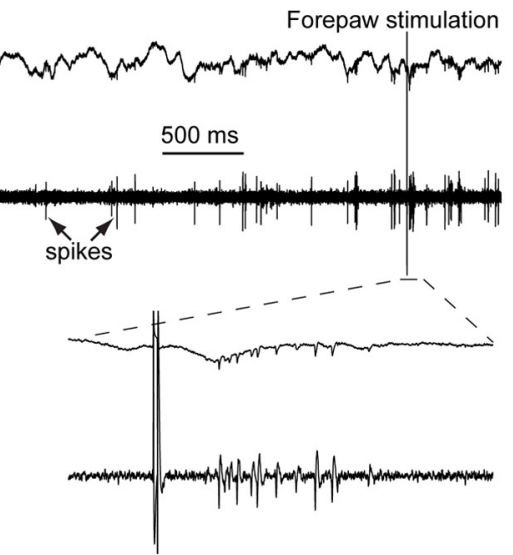

C
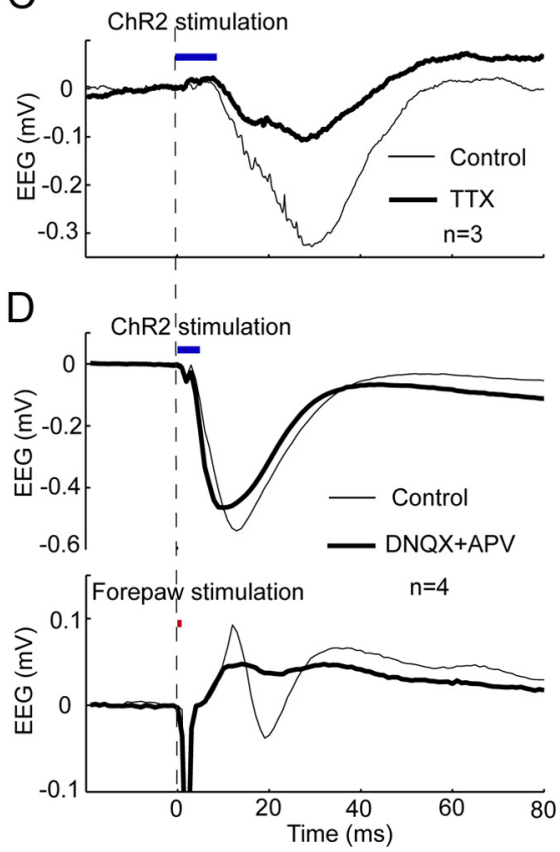

Figure 3. Role of spiking and synaptic activity in ChR2-mediated responses. A, LFP responses (top) during ChR2 or forepaw stimulation filtered to resolve MUA (middle). Representative spikes are indicated with arrows. Two segments of ChR2 or forepaw stimulation response are shown in enlarged views (bottom). $\boldsymbol{B}$, Quantification of spiking with $5 \mathrm{~ms}$ of laser stimulation. Top, Raster plot of spikes during 20 trials and average spike waveform (example mouse shown). Bottom, Plot of spiking rate with $5 \mathrm{~ms}$ bin size ( $n=4$ mice). The shaded area indicates the SEM. C, Effect of locally applied TTX $30 \mu$ m on ChR2-mediated EEG responses; average response from $n=3$ mice is shown. $\boldsymbol{D}$, Antagonists of glutamate receptors (DNQX $200 \mu \mathrm{m}$ and APV $500 \mu \mathrm{m}$ ) block sensorymediated, but not ChR2-mediated, EEG response; average from $n=4$ mice is shown.

stronger forepaw stimulation (2 ms), we found greater recovery of the forepaw-evoked LFP response $2 \mathrm{~h}$ after reperfusion (77 \pm $21 \%$ of baseline level, $n=4, p=0.36$ ), consistent with previous data examining sensory maps (Murphy et al., 2008). Using stronger sensory stimulation, we were also able to better match the amplitude of the optogenetic and sensory responses $(0.67 \pm 0.19$ $\mathrm{mV}$ optogenetic vs $0.52 \pm 0.07 \mathrm{mV}$ forepaw, $n=4, p=0.53)$, and confirmed that the sensory response was consistently depressed at $30 \mathrm{~min}$ after reperfusion ( $36 \pm 7 \%$ of baseline level, $n=4, p<$ 0.01 ), while the optogenetic response recovered to $103 \pm 5 \%$ of baseline level at $30 \mathrm{~min}(n=4, p=0.60)$.

To investigate the nature of the ChR2-evoked response, we performed additional LFP analysis and parallel pharmacological experiments. Data obtained from all 16 channels were bandpass filtered $(600-6000 \mathrm{~Hz})$ to isolate spike activity that spontaneously occurred or was evoked in response to ChR2 or sensory stimulation (Fig. $3 A, B)$. Despite the laser entering at the brain surface, we detected an

increase in spikes evoked by $\mathrm{ChR} 2$ stimulation from electrodes within layer 5. Although $\mathrm{ChR} 2$ is expressed at highest density within layer 5 neuron cell bodies, laser stimulation is likely to predominantly activate superficial dendritic tufts. The spiking is consistent with a direct activation of ChR2expressing neurons (Kahn et al., 2011). Consistent with a component of lightstimulated signals being dependent on action potentials, locally applied TTX led to a $67 \%$ reduction in ChR2-evoked EEG signal (Fig. 3C). To assess the contribution of synaptic transmission, we applied DNQX and APV locally and observed a complete blockade of the depolarizing forepaw-evoked signal, but no significant effect on the ChR2evoked peak EEG response (Fig. 3D).

\section{Spatiotemporal evolution of ischemic depolarization and relation to ChR2-mediated responses}

Changes in cortical absorbance triggered by ischemic depolarization can be detected using IOS imaging (Obeidat et al., 2000; Jarvis et al., 2001; Murphy et al., 2008). IOSs were acquired to examine the relationship between ischemic depolarization and excitability as assayed by ChR2 stimulation and EEG recording (Fig. $4 A$ ). When the spreading ischemic depolarization IOS wave propagated to the ChR2-stimulated cortex [Fig. $4 B, C$, ROI 2 (R2)], the EEG response was rapidly reduced. IOS imaging showed that the ischemic depolarization wave started as an increase in scattering (Fig. 4C, white) from the most anterior and lateral portions of the imaged cortex and spread posteriorly and medially (Murphy et al., 2008). At the $140 \mathrm{~s}$ time point, the maximum change of IOS occurred in R2 (Fig. $4 B, C)$ and was coincident with the maximally attenuated ChR2-mediated EEG response (Fig. 4A). Analysis of group data $(n=7$ mice) confirmed the coincidence between local ischemic depolarization and the loss of excitability with a temporal precision of $5 \pm 1 \mathrm{~s}$.

\section{Discussion}

ChR2-based assessment of neuronal excitability during global ischemia

Preservation of neuronal excitability is critical for functional recovery after stroke (Bütefisch et al., 2003; Murphy and Corbett, 2009; Clarkson et al., 2010). Previous data have shown that synaptic transmission is depressed during ischemia and following reperfusion (Xu and Pulsinelli, 1996; Gao et al., 1998). This effect can be measured using cortical EEG recordings (Astrup et al., 1981) that presumably reflect synaptic activity. However, it has been a challenge to directly assess neuronal excitability in vivo while EEG activity is depressed during ischemia and within $1 \mathrm{~h}$ after reperfusion. Conven- 
A

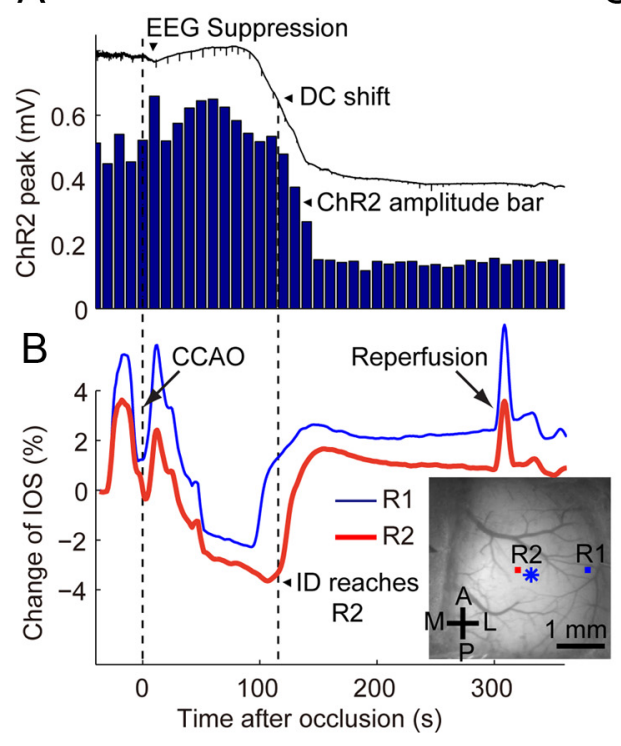

C
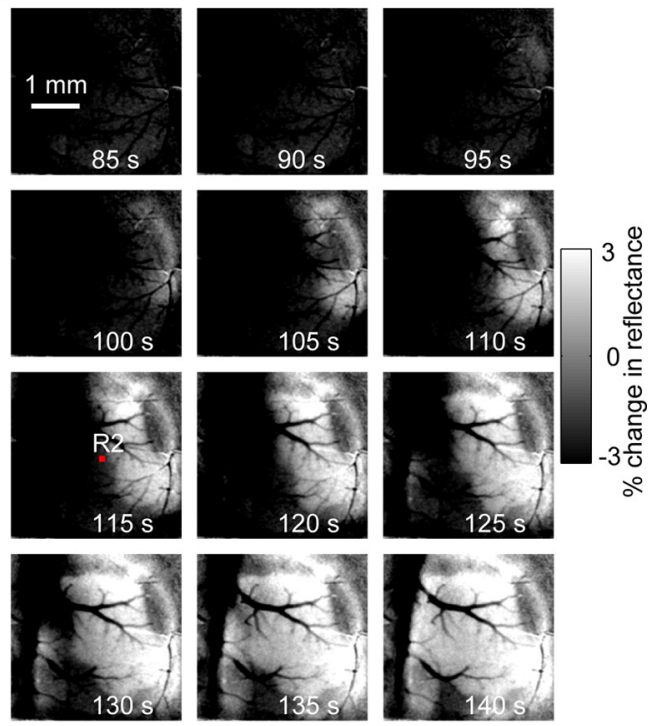

Figure 4. IOS imaging indicates that the ChR2-mediated EEG response is suppressed coincidently with the DC shift during global ischemia. $\boldsymbol{A}$, Bar plot of ChR2-mediated peakEEG amplitude shows transient increase after spontaneous EEG suppression, but before the DC potential depolarized. When the DC potential indicated peak ischemic depolarization, the ChR2-mediated EEG amplitude decreased to minimal values. $\boldsymbol{B}$, Ischemic depolarization (ID) wave characterized by a rapid increase in IOS signal. Five-point smoothed time plots for different regions of interest (R1 and R2, shown in the inset) indicate the propagation of the ischemic depolarization wave. R1 is close to the EEG recording electrode. R2 is from a site near laser stimulation (indicated by asterisk). Part of the time courses between -20 and $50 \mathrm{~s}$ were affected by motion artifacts associated with the CCA0 procedure. C, Differential images of intrinsic signal show the spatiotemporal evolution of the ischemic depolarization wave. The ischemic depolarization wave starts typically as an increase in light scattering (shown as white) from the most anterior and lateral portions of the imaged cortex and spreads posteriorly and medially. When the IOS response peak is present at site R2 (at $140 \mathrm{~s}$, see $\boldsymbol{B}$ and $\boldsymbol{C}$ ), the $\mathrm{ChR2}$ peak amplitude was reduced to a minimum value in $\boldsymbol{A}$.

tional recording of sensation-evoked potentials has revealed hyperexcitability from time points $1-6 \mathrm{~h}$ after focal ischemia (Fujioka et al., 2004). Neuronal excitability can be assessed indirectly in vivo after stroke or trauma using paired-pulse sensory stimulation (Fujioka et al., 2004; Ding et al., 2011). However, previous work has shown that the cortical sensoryevoked potential is abolished after global ischemia (Lesnick et al., 1986). We have used ChR2 to directly depolarize neurons and extend the previous measurement of cortical excitability with embedded stimulating electrodes (Lesnick et al., 1986). We expect ChR2 to be predominantly expressed in layer 5 cortical pyramidal neurons (Arenkiel et al., 2007; Wang et al., 2007; Ayling et al., 2009). Therefore, the changes in neuronal excitability we observe during acute phases of global ischemia are relatively specific to these neurons. With optogenetics, we show that it would be feasible to study the excitability of select cell populations, such as large aspiny neurons (Pang et al., 2002; Li et al., 2009) or GABAergic interneurons (Redecker et al., 2002; Boyden et al., 2005; Clarkson et al., 2010), during ischemia.

The global ischemia model used here is clinically related to cardiac arrest or cardiac surgery (Hofmeijer and van Putten, 2012). Although this model does not recapitulate more clinically relevant middle cerebral artery occlusion models (Dirnagl et al., 1999), we can precisely control the duration of ischemia to within seconds and can rapidly alternate between the initiation of ischemia or reperfusion. The methods used here could be extended to a focal ischemia model, but it would be difficult to monitor excitability within the first minutes of occlusion since these models are not necessarily compatible with continuous recording ( $\mathrm{Li}$ and Murphy, 2008). Since it is possible to perform scanning stimulation (Ayling et al., 2009), we may be able to complete a spatial optogenetic analysis of a focal photothrombotic model (Mohajerani et al., 2011). This would distinguish changes in excitabil- ity within specific regions such as the infarct, penumbra, and normal tissue (Chen et al., 2006b). Additionally, it would be possible to study neuronal excitability with the recently developed hippocampal ischemia model (Barth and Mody, 2011) using in vivo fiber optic activation of ChR2. Given its wide applications, ChR2-based assessment of neuronal excitability could be a mouse analog of transcranial magnetic stimulation, providing background for translational stroke studies (Bütefisch et al., 2003).

The nature of the ChR2-mediated electrophysiological signal The ChR2-mediated EEG or LFP response can reflect both direct ChR2-mediated and indirect synaptic activation, and can last longer than the duration of the light stimulus (Harrison et al., 2012; Lim et al., 2012). Given the relatively long deactivation time for ChR2 (Fenno et al., 2011), even the response to direct activation should be on the order of $10-15 \mathrm{~ms}$ in duration, consistent with the peak responses we observe. Some of the indirect effects from light stimulation may be attributed to ChR2 directly gating channels on axons that produce neurotransmitter release (Petreanu et al., 2007; Schoenenberger et al., 2011). However, the initial peak component of the ChR2-mediated response (within $10-20 \mathrm{~ms}$ ) was relatively insensitive to glutamate receptor antagonists and is likely derived from direct channel activation and secondary action potential firing given its sensitivity to tetrodotoxin. Therefore, ChR2-mediated peak EEG or LFP signals may predominantly reflect subthreshold activity and poorly synchronized firing among populations of neurons.

Selective deficits in sensory processing following reperfusion in mouse cortex

Previous studies have shown that ischemia can profoundly change synaptic transmission and neuronal excitability (Pang et al., 2002; Fujioka et al., 2004; Zhang et al., 2006; Liang et al., 
2009; Clarkson et al., 2010; Paz et al., 2010). However, the results were not always consistent between models. Using assessment of forepaw stimulation-evoked cortical potentials, we find that forepaw sensation-dependent cortical synaptic transmission was inhibited even $1 \mathrm{~h}$ after reperfusion from CCAO. This finding was consistent with previous work (Astrup et al., 1981; Murphy et al., 2008) and may reflect a persistent synaptic transmission defect (Bolay and Dalkara, 1998). In contrast to synaptic responses, the use of ChR2 stimulation revealed that neuronal excitability was recovered with reperfusion. These results suggest that the selective deficits in sensory processing following reperfusion in mouse cortex are not necessarily attributed to a loss of direct neuronal excitability. The absence of forepaw-evoked and spontaneous EEG or LFP responses is indicative of a loss of synaptic activity. This fact, combined with the ability to depolarize neurons with light in ChR2 transgenic mice, argues for a long-term selective block in synaptic transmission during the postreperfusion period.

The mismatches between changes within sensory processing and neuronal excitability after transient global ischemia may be due to a block of conduction within white matter (including thalamus) (Lesnick et al., 1986). The short-latency forepaw response (within $20 \mathrm{~ms}$ of forepaw stimulation) we quantified is likely to be directly related to thalamocortical activity (Wu et al., 2012). Further research may focus on functional changes within thalamocortical projections during stroke (Paz et al., 2010) by performing direct thalamic recordings (Ding et al., 2011). Moreover, it is also possible that postsynaptic cortical mechanisms are involved in the ischemia-induced loss of synaptic activity such as glutamate receptor endocytosis (Man et al., 2000).

\section{Ischemic depolarization is associated with a loss of neuronal excitability}

Previous work has shown that ischemic depolarization is the major trigger of neuronal structural damage (Obeidat et al., 2000; Murphy et al., 2008; Risher et al., 2010) during stroke. Here we show that ischemic depolarization is directly related to the loss of neuronal excitability measured by ChR2-mediated EEG responses. Ischemic depolarization is initiated at the edge of the ischemic core by elevated $\left[\mathrm{K}^{+}\right]_{\mathrm{o}}$ and glutamate (Nedergaard, 1996; Lee et al., 1999; Strong and Dardis, 2005). Since both $\mathrm{K}^{+}$ and glutamate may excite neurons, this could be why we observed a transient increase in neuronal excitability within 1 min after CCAO. In our experiments, 5 min of CCAO would induce a DC shift of $\sim 10$ min in duration, about three times longer than normoxic spreading depression (Leao, 1944) induced in healthy brain. The prolonged collapse of ion gradients during ischemic depolarization could initiate a cascade of damaging events (Dirnagl et al., 1999) that lead to prolonged inhibition of forepaw sensory responses. The dependence of ChR2 responses on an intact resting membrane potential (Nagel et al., 2003) is consistent with its strong inhibition during ischemic depolarization and rapid recovery during reperfusion.

Although the DC potential did not completely return to baseline levels after reperfusion, the ChR2 peak amplitude recovered nearly fully when compared with the ischemic depolarization process. It is possible that this reflects hyperexcitability that is observed after stroke, which enhances the ChR2 response despite incomplete recovery of membrane potential (Fujioka et al., 2004). This may be related to a reduction in $\mathrm{GABA}_{\mathrm{A}}$ receptor expression after stroke (Schiene et al., 1996; Redecker et al., 2002). More work is also needed to determine whether $\mathrm{GABA}_{\mathrm{A}}$ receptor-mediated tonic inhibition plays a role (Clarkson et al., 2010).

In both experimental and clinical studies, recurring ischemic depolarization or spreading depression waves have been associated with an exacerbation of damage after stroke (Dirnagl et al., 1999; Jarvis et al., 2001; Dreier et al., 2006; Shin et al., 2006; Strong et al., 2007; Risher et al., 2010). In vitro data indicate that ischemic depolarization can alter the balance between excitation and inhibition (Berger et al., 2008). Our study supports the idea that agents that reduce ischemic depolarization are potential therapeutic targets for stroke (Douglas et al., 2011; Risher et al., 2011). Reducing ischemic depolarization will help to maintain excitability and may prevent prolonged loss of sensory processing, leading to better outcomes following reperfusion.

In summary, our results indicate that neuronal excitability recovers much faster than sensory-evoked synaptic activity in vivo following ischemia and reperfusion. This delineates a time window following ischemia where synaptically silent, yet excitable neurons are present. We anticipate that optogenetically stimulated ChR2 will allow investigators to further examine the excitability of unique cell populations during ischemic damage and reperfusion.

\section{References}

Aravanis AM, Wang LP, Zhang F, Meltzer LA, Mogri MZ, Schneider MB, Deisseroth K (2007) An optical neural interface: in vivo control of rodent motor cortex with integrated fiberoptic and optogenetic technology. J Neural Eng 4:S143-S156.

Arenkiel BR, Peca J, Davison IG, Feliciano C, Deisseroth K, Augustine GJ, Ehlers MD, Feng G (2007) In vivo light-induced activation of neural circuitry in transgenic mice expressing channelrhodopsin-2. Neuron 54:205-218.

Astrup J, Siesjö BK, Symon L (1981) Thresholds in cerebral ischemia-the ischemic penumbra. Stroke 12:723-725.

Ayling OG, Harrison TC, Boyd JD, Goroshkov A, Murphy TH (2009) Automated light-based mapping of motor cortex by photoactivation of channelrhodopsin-2 transgenic mice. Nat Methods 6:219-224.

Ba AM, Guiou M, Pouratian N, Muthialu A, Rex DE, Cannestra AF, Chen JW, Toga AW (2002) Multiwavelength optical intrinsic signal imaging of cortical spreading depression. J Neurophysiol 88:2726-2735.

Barth AM, Mody I (2011) Changes in hippocampal neuronal activity during and after unilateral selective hippocampal ischemia in vivo. J Neurosci 31:851-860.

Berger M, Speckmann EJ, Pape HC, Gorji A (2008) Spreading depression enhances human neocortical excitability in vitro. Cephalalgia 28:558-562.

Bokil H, Andrews P, Kulkarni JE, Mehta S, Mitra PP (2010) Chronux: a platform for analyzing neural signals. J Neurosci Methods 192:146-151.

Bolay H, Dalkara T (1998) Mechanisms of motor dysfunction after transient MCA occlusion: persistent transmission failure in cortical synapses is a major determinant. Stroke 29:1988-1993.

Boyden ES, Zhang F, Bamberg E, Nagel G, Deisseroth K (2005) Millisecondtimescale, genetically targeted optical control of neural activity. Nat Neurosci 8:1263-1268.

Bütefisch CM, Netz J, Wessling M, Seitz RJ, Hömberg V (2003) Remote changes in cortical excitability after stroke. Brain 126:470-481.

Chen S, Li P, Luo W, Gong H, Zeng S, Luo Q (2006a) Time-varying spreading depression waves in rat cortex revealed by optical intrinsic signal imaging. Neurosci Lett 396:132-136.

Chen S, Feng Z, Li P, Jacques SL, Zeng S, Luo Q (2006b) In vivo optical reflectance imaging of spreading depression waves in rat brain with and without focal cerebral ischemia. J Biomed Opt 11:34002

Clarkson AN, Huang BS, Macisaac SE, Mody I, Carmichael ST (2010) Reducing excessive GABA-mediated tonic inhibition promotes functional recovery after stroke. Nature 468:305-309.

Crunelli V, Hughes SW (2010) The slow $(<1 \mathrm{~Hz})$ rhythm of non-REM sleep: a dialogue between three cardinal oscillators. Nat Neurosci 13:9-17. 
Ding MC, Wang Q, Lo EH, Stanley GB (2011) Cortical excitation and inhibition following focal traumatic brain injury. J Neurosci 31:14085-14094.

Dirnagl U, Iadecola C, Moskowitz MA (1999) Pathobiology of ischaemic stroke: an integrated view. Trends Neurosci 22:391-397.

Douglas HA, Callaway JK, Sword J, Kirov SA, Andrew RD (2011) Potent inhibition of anoxic depolarization by the sodium channel blocker dibucaine. J Neurophysiol 105:1482-1494.

Dreier JP, Woitzik J, Fabricius M, Bhatia R, Major S, Drenckhahn C, Lehmann TN, Sarrafzadeh A, Willumsen L, Hartings JA, Sakowitz OW, Seemann JH, Thieme A, Lauritzen M, Strong AJ (2006) Delayed ischaemic neurological deficits after subarachnoid haemorrhage are associated with clusters of spreading depolarizations. Brain 129:3224-3237.

Fenno L, Yizhar O, Deisseroth K (2011) The development and application of optogenetics. Annu Rev Neurosci 34:389-412.

Fujioka H, Kaneko H, Suzuki SS, Mabuchi K (2004) Hyperexcitabilityassociated rapid plasticity after a focal cerebral ischemia. Stroke 35:e346-e348.

Gao TM, Howard EM, Xu ZC (1998) Transient neurophysiological changes in CA3 neurons and dentate granule cells after severe forebrain ischemia in vivo. J Neurophysiol 80:2860-2869.

Harrison TC, Sigler A, Murphy TH (2009) Simple and cost-effective hardware and software for functional brain mapping using intrinsic optical signal imaging. J Neurosci Methods 182:211-218.

Harrison TC, Ayling OG, Murphy TH (2012) Distinct cortical circuit mechanisms for complex forelimb movement and motor map topography. Neuron 74:397-409.

Hofmeijer J, van Putten MJ (2012) Ischemic cerebral damage: an appraisal of synaptic failure. Stroke 43:607-615.

Hossmann KA (2006) Pathophysiology and therapy of experimental stroke. Cell Mol Neurobiol 26:1057-1083.

Jarvis CR, Anderson TR, Andrew RD (2001) Anoxic depolarization mediates acute damage independent of glutamate in neocortical brain slices. Cereb Cortex 11:249-259.

Kahn I, Desai M, Knoblich U, Bernstein J, Henninger M, Graybiel AM, Boyden ES, Buckner RL, Moore CI (2011) Characterization of the functional MRI response temporal linearity via optical control of neocortical pyramidal neurons. J Neurosci 31:15086-15091.

Kravitz AV, Freeze BS, Parker PR, Kay K, Thwin MT, Deisseroth K, Kreitzer AC (2010) Regulation of parkinsonian motor behaviours by optogenetic control of basal ganglia circuitry. Nature 466:622-626.

Leao AAP (1944) Spreading depression of activity in the cerebral cortex. J Neurophysiol 7:359-390.

Lee JM, Zipfel GJ, Choi DW (1999) The changing landscape of ischaemic brain injury mechanisms. Nature 399:A7-A14.

Lesnick JE, Coyer PE, Michele JJ, Welsh FA, Simeone FA (1986) Comparison of the somatosensory evoked potential and the direct cortical response following severe incomplete global ischemia: selective vulnerability of the white matter conduction pathways. Stroke 17:1247-1250.

Liang R, Pang ZP, Deng P, Xu ZC (2009) Transient enhancement of inhibitory synaptic transmission in hippocampal CA1 pyramidal neurons after cerebral ischemia. Neuroscience 160:412-418.

Li P, Murphy TH (2008) Two-photon imaging during prolonged middle cerebral artery occlusion in mice reveals recovery of dendritic structure after reperfusion. J Neurosci 28:11970-11979.

Li Y, Lei Z, Xu ZC (2009) Enhancement of inhibitory synaptic transmission in large aspiny neurons after transient cerebral ischemia. Neuroscience 159:670-681.

Lim DH, Mohajerani MH, Ledue J, Boyd J, Chen S, Murphy TH (2012) In vivo large-scale cortical mapping using channelrhodopsin-2 stimulation in transgenic mice reveals asymmetric and reciprocal relationships between cortical areas. Front Neural Circuits 6:11.

Liu RR, Murphy TH (2009) Reversible cyclosporin A-sensitive mitochondrial depolarization occurs within minutes of stroke onset in mouse somatosensory cortex in vivo: a two-photon imaging study. J Biol Chem 284:36109-36117.

Man HY, Lin JW, Ju WH, Ahmadian G, Liu L, Becker LE, Sheng M, Wang YT (2000) Regulation of AMPA receptor-mediated synaptic transmission by clathrin-dependent receptor internalization. Neuron 25:649-662.

Miesenböck G (2009) The optogenetic catechism. Science 326:395-399.

Mohajerani MH, McVea DA, Fingas M, Murphy TH (2010) Mirrored bilat- eral slow-wave cortical activity within local circuits revealed by fast bihemispheric voltage-sensitive dye imaging in anesthetized and awake mice. J Neurosci 30:3745-3751.

Mohajerani MH, Aminoltejari K, Murphy TH (2011) Targeted ministrokes produce changes in interhemispheric sensory signal processing that are indicative of disinhibition within minutes. Proc Natl Acad Sci U S A 108:E183-E191.

Mostany R, Portera-Cailliau C (2011) Absence of large-scale dendritic plasticity of layer 5 pyramidal neurons in peri-infarct cortex. J Neurosci 31:1734-1738.

Murphy TH (2011) Two-photon imaging of neuronal structural plasticity in mice during and after ischemia. In: Imaging in neuroscience: a laboratory mannual, Ed 1 (Helmchen F, Konnerth A, eds). New York: Cold Spring Harbor Laboratory.

Murphy TH, Corbett D (2009) Plasticity during stroke recovery: from synapse to behaviour. Nat Rev Neurosci 10:861-872.

Murphy TH, Li P, Betts K, Liu R (2008) Two-photon imaging of stroke onset in vivo reveals that NMDA-receptor independent ischemic depolarization is the major cause of rapid reversible damage to dendrites and spines. J Neurosci 28:1756-1772.

Nagel G, Szellas T, Huhn W, Kateriya S, Adeishvili N, Berthold P, Ollig D, Hegemann P, Bamberg E (2003) Channelrhodopsin-2, a directly lightgated cation-selective membrane channel. Proc Natl Acad Sci U S A 100:13940-13945.

Nedergaard M (1996) Spreading depression as a contributor to ischemic brain damage. Adv Neurol 71:75-83.

Obeidat AS, Jarvis CR, Andrew RD (2000) Glutamate does not mediate acute neuronal damage after spreading depression induced by $\mathrm{O} 2 /$ glucose deprivation in the hippocampal slice. J Cereb Blood Flow Metab 20:412-422.

Pang ZP, Deng P, Ruan YW, Xu ZC (2002) Depression of fast excitatory synaptic transmission in large aspiny neurons of the neostriatum after transient forebrain ischemia. J Neurosci 22:10948-10957.

Paz JT, Christian CA, Parada I, Prince DA, Huguenard JR (2010) Focal cortical infarcts alter intrinsic excitability and synaptic excitation in the reticular thalamic nucleus. J Neurosci 30:5465-5479.

Petreanu L, Huber D, Sobczyk A, Svoboda K (2007) Channelrhodopsin-2assisted circuit mapping of long-range callosal projections. Nat Neurosci 10:663-668.

Quiroga RQ, Nadasdy Z, Ben-Shaul Y (2004) Unsupervised spike detection and sorting with wavelets and superparamagnetic clustering. Neural Comput 16:1661-1687.

Redecker C, Wang W, Fritschy JM, Witte OW (2002) Widespread and longlasting alterations in GABA(A)-receptor subtypes after focal cortical infarcts in rats: mediation by NMDA-dependent processes. J Cereb Blood Flow Metab 22:1463-1475.

Risher WC, Ard D, Yuan J, Kirov SA (2010) Recurrent spontaneous spreading depolarizations facilitate acute dendritic injury in the ischemic penumbra. J Neurosci 30:9859-9868.

Risher WC, Lee MR, Fomitcheva IV, Hess DC, Kirov SA (2011) Dibucaine mitigates spreading depolarization in human neocortical slices and prevents acute dendritic injury in the ischemic rodent neocortex. PLoS One 6:e22351.

Schiene K, Bruehl C, Zilles K, Qü M, Hagemann G, Kraemer M, Witte OW (1996) Neuronal hyperexcitability and reduction of GABAA-receptor expression in the surround of cerebral photothrombosis. J Cereb Blood Flow Metab 16:906-914.

Schoenenberger P, Schärer YP, Oertner TG (2011) Channelrhodopsin as a tool to investigate synaptic transmission and plasticity. Exp Physiol 96:34-39.

Scott NA, Murphy TH (2012) Hemodynamic responses evoked by neuronal stimulation via channelrhodopsin-2 can be independent of intracortical glutamatergic synaptic transmission. PLoS One 7:e29859.

Shin HK, Dunn AK, Jones PB, Boas DA, Moskowitz MA, Ayata C (2006) Vasoconstrictive neurovascular coupling during focal ischemic depolarizations. J Cereb Blood Flow Metab 26:1018-1030.

Strong AJ, Dardis R (2005) Depolarisation phenomena in traumatic and ischaemic brain injury. Adv Tech Stand Neurosurg 30:3-49.

Strong AJ, Anderson PJ, Watts HR, Virley DJ, Lloyd A, Irving EA, Nagafuji T, Ninomiya M, Nakamura H, Dunn AK, Graf R (2007) Peri-infarct depo- 
larizations lead to loss of perfusion in ischaemic gyrencephalic cerebral cortex. Brain 130:995-1008.

Tran S, Chen S, Liu RR, Xie Y, Murphy TH (2012) Moderate or deep local hypothermia does not prevent the onset of ischemia-induced dendritic damage. J Cereb Blood Flow Metab 32:437-442.

Wang H, Peca J, Matsuzaki M, Matsuzaki K, Noguchi J, Qiu L, Wang D, Zhang F, Boyden E, Deisseroth K, Kasai H, Hall WC, Feng G, Augustine GJ (2007) High-speed mapping of synaptic connectivity using photostimulation in channelrhodopsin-2 transgenic mice. Proc Natl Acad Sci U S A 104:8143-8148.

Wu D, Xiong W, Jia X, Geocadin RG, Thakor NV (2012) Short- and longlatency somatosensory neuronal responses reveal selective brain injury and effect of hypothermia in global hypoxic ischemia. J Neurophysiol 107:1164-1171.

Xu ZC, Pulsinelli WA (1994) Responses of CA1 pyramidal neurons in rat hippocampus to transient forebrain ischemia: an in vivo intracellular recording study. Neurosci Lett 171:187-191.

Xu ZC, Pulsinelli WA (1996) Electrophysiological changes of CA1 pyramidal neurons following transient forebrain ischemia: an in vivo intracellular recording and staining study. J Neurophysiol 76:16891697.

Zhang F, Wang LP, Brauner M, Liewald JF, Kay K, Watzke N, Wood PG, Bamberg E, Nagel G, Gottschalk A, Deisseroth K (2007) Multimodal fast optical interrogation of neural circuitry. Nature 446:633-639.

Zhang S, Boyd J, Delaney K, Murphy TH (2005) Rapid reversible changes in dendritic spine structure in vivo gated by the degree of ischemia. J Neurosci 25:5333-5338.

Zhang Y, Deng P, Li Y, Xu ZC (2006) Enhancement of excitatory synaptic transmission in spiny neurons after transient forebrain ischemia. J Neurophysiol 95:1537-1544. 